\title{
INTERNET ET SANTÉ DES MINORITÉS SEXUELLES AU CANADA : UNE ÉTUDE EXPLORATOIRE
}

\author{
Joseph Lévy, Jean Dumas, Christine Thoër, Bill Ryan, Alain Léobon
}

\author{
S.F.S.P. | « Santé Publique »
}

2009/hs2 Vol. 21 | pages 53 à 63

ISSN 0995-3914

Article disponible en ligne à l'adresse :

https://www.cairn.info/revue-sante-publique-2009-hs2-page-53.htm

Distribution électronique Cairn.info pour S.F.S.P..

(C) S.F.S.P.. Tous droits réservés pour tous pays.

La reproduction ou représentation de cet article, notamment par photocopie, n'est autorisée que dans les limites des conditions générales d'utilisation du site ou, le cas échéant, des conditions générales de la licence souscrite par votre établissement. Toute autre reproduction ou représentation, en tout ou partie, sous quelque forme et de quelque manière que ce soit, est interdite sauf accord préalable et écrit de l'éditeur, en dehors des cas prévus par la législation en vigueur en France. Il est précisé que son stockage dans une base de données est également interdit. 


\title{
Internet et santé des minorités sexuelles au Canada : une étude exploratoire
}

\author{
Internet and the health of sexual minorities in Canada: \\ an exploratory study
}

\author{
Joseph Lévy (1), Jean Dumas (2), Christine Thoër (3), Bill Ryan (4), Alain Léobon (5)
}

Résumé : Les organismes œuvrant auprès des minorités LGBT (personnes se définissant comme lesbiennes, gaies, bisexuelles, bispirituelles ou transgenres) sont de plus en plus nombreux à développer des sites internet et à recourir aux stratégies d'intervention en ligne visant l'information, la prévention et l'amélioration de la santé et de la qualité de vie de ces populations. Afin de dégager un portrait canadien dans ce domaine, une étude a été menée auprès d'organismes qui ont répondu à un questionnaire portant sur leurs pratiques associées à l'internet. Les résultats mettent en évidence les multiples avantages que les organismes communautaires associent à ce mode d'intervention mais soulignent aussi les contraintes auxquelles ils font face et les difficultés que peut poser la mise en place d'intervention en ligne.

Mots-clés : Minorités sexuelles - internet - Canada - santé - intervention en ligne.

Summary: A large number of organizations working with LGBT minorities are currently in the process of developing websites and using online intervention strategies with a view to providing information, promoting prevention and improving healthcare and quality of life. This paper outlines the findings of a study conducted among organizations that responded to a survey on their internet activities and interventions, and aims to provide a national Canadian profile of the field. The results of this survey highlight the many advantages which community organizations tend to associate with this type of intervention. But they also underline the constraints which such organizations face and the difficulties that may arise as a result of developing online interventions.

Keywords: Sexual minorities - Internet - Canada - health - online intervention.

(1) Professeur, département de sexologie, UQAM, Équipe de recherche Sexualités et genres : Vulnérabilité, Résilience.

(2) Doctorant, département de communication sociale et publique, UQAM, Équipe de recherche Sexualités et genres : Vulnérabilité, Résilience.

(3) Professeure, département de communication sociale et publique, UQAM, Équipe de recherche Sexualités et genres: Vulnérabilité, Résilience.

(4) Professeur, École de travail social, Université McGill, Équipe de recherche Sexualités et genres: Vulnérabilité, Résilience.

(5) Collaborateur et professeur associé, Centre national de recherche scientifique, France, Équipe de recherche Sexualités et genres : Vulnérabilité, Résilience. 


\section{Introduction}

L'évaluation de la santé des minorités sexuelles LGBT (personnes se définissant comme lesbiennes, gaies, bisexuelles, bispirituelles ou transgenres [34]) constitue une préoccupation importante dans le champ de la santé publique comme en témoigne la multiplication des ouvrages sur cette question [25]. Parallèlement, les stratégies développées dans l'intervention auprès de ces populations tendent à se diversifier [34] et à prendre en compte la contribution des nouvelles technologies de communication. L'internet offre en effet un ensemble d'outils informationnels et interactifs de plus en plus utilisés par la population générale [30] et par les minorités sexuelles [23]. Aussi, les organismes œuvrant auprès des minorités sexuelles sont-ils de plus en plus nombreux à recourir à ces outils afin de mieux répondre aux besoins spécifiques de ces populations. Dans cette perspective, et à partir des résultats préliminaires d'une recherche en cours, l'article propose une première analyse des interventions de santé publique réalisées à l'aide de l'internet auprès des minorités sexuelles au Canada.

\section{Enjeux de santé des minorités sexuelles et potentiel de l'internet}

Les recherches menées depuis la fin des années 1970 ont suggéré des prévalences élevées de plusieurs problèmes de santé parmi les LGBT. À part les répercussions du $\mathrm{VIH} /$ sida et des autres infections transmissibles sexuellement et par le sang (ITSS), qui ont durement touché les hommes ayant des relations sexuelles avec d'autres hommes (HARSAH) depuis le début de l'épidémie $[28,1,20]$, d'autres problématiques de santé sont aussi préoccupantes : l'anxiété et la dépression, les idéations suicidaires et les tentatives de suicide $[29,27,9,24,25]$, le tabagisme et la consommation d'alcool et de drogues $[25,32,36,38]$ et certains cancers, comme celui du sein parmi les lesbiennes [19]. Les enjeux entourant l'acceptation personnelle, familiale et sociale des orientations sexuelles, liés à l'homophobie et la stigmatisation peuvent aussi influer sur la santé mentale [5, 8]. Au Canada, selon l'Enquête sur la santé dans les collectivités canadiennes [37], les minorités sexuelles ont déclaré des niveaux de troubles de l'humeur ou de troubles anxieux supérieurs à ceux de la population hétérosexuelle.

Les recherches suggèrent également que des inégalités sont présentes dans l'accès aux services de santé, les minorités sexuelles étant plus nombreuses à déclarer des besoins d'information et de soins de santé non satisfaits ou à être confrontées à des attitudes renvoyant à de l'homophobie et à de l'hétérosexisme de la part des professionnels de la santé [3, 10, 33, 35, 37, 31].

Face à ces difficultés, et en particulier, dans le contexte de l'épidémie du $\mathrm{VIH} /$ sida, des organismes communautaires ont décidé d'utiliser l'internet dans le cadre de leurs interventions de prévention, en proposant notamment des sites offrant des informations (description des agents pathogènes associés aux ITSS, des niveaux de risques associés aux pratiques sexuelles, répertoires de ressources et de professionnels de la santé, etc.) mais aussi en développant des programmes de prévention sur les sites de rencontre entre hommes, dans les salons de clavardage (chat rooms) ou sur les 
babillards (tableaux d'affichage questions-réponses) [7, 26]. Ces initiatives de prévention ont recours à une équipe d'intervenants, qui assure une présence dans ces espaces pour répondre aux questions et discuter de la prévention des ITSS ou d'autres questions relatives à la santé sexuelle.

Ces outils sont particulièrement intéressants pour rejoindre les populations LGBT résidant hors des grands centres urbains, les jeunes et ceux qui ne fréquentent pas les lieux de rencontre et de socialisation urbains au sein desquels sont concentrées la plupart des activités de prévention des ITSS et du VIH/sida $[16,14,22]$. La garantie d'anonymat constitue une caractéristique qui semble valorisée, comme le soulignent des recherches rapportant que des internautes préfèrent recourir à l'internet plutôt qu'aux professionnels de la santé ou au soutien de leur entourage, par crainte de subir des formes de stigmatisation ou de discrimination [12, 13, 21].

Si le potentiel de l'internet pour les interventions auprès des minorités sexuelles semble évident, la recherche a essentiellement porté sur les programmes destinés aux HARSAH, tandis que ceux orientés vers d'autres groupes sont très peu documentés. L'article présente une description et une première analyse des sites et autres sources d'information destinées à l'ensemble des minorités sexuelles au Canada à partir des résultats préliminaires d'une recherche menée en deux étapes. Dans un premier temps, une recension des sites canadiens proposant des informations sur la santé aux personnes LGBT a été réalisée. Puis des questionnaires ont été acheminés (6) aux différentes organisations recensées dans le but de mieux documenter leurs caractéristiques et de cerner avec précision leurs activités de prévention en ligne. Il s'agit là de la première étude réalisée dans le contexte canadien et considérant l'ensemble des minorités sexuelles.

\section{Méthodologie}

Pour effectuer la recension des sites canadiens [14,6], ceux-ci ont été repérés entre 2007 et 2009 avec des moteurs de recherche en ligne et à l'aide de mots-clés (par exemple : santé, LGBT, sexualité, etc.). Les sites retenus devaient être gérés par des organismes situés au Canada et proposer des informations touchant la santé des minorités sexuelles. Le recoupement entre les nombreux sites identifiés et ceux présentés dans différents répertoires disponibles en ligne permet de conclure à une certaine représentativité de l'échantillon ainsi constitué(7). Pour compléter les informations recueillies sur les sites, une seconde étape de la recherche invitait les gestionnaires de ces sites à participer à un questionnaire en ligne portant sur la mission de l’organisme, les activités déployées sur l'internet et

(6) En mars 2008, un groupe focus réunissant des gestionnaires d'organismes provenant de plusieurs régions du pays et ayant développé des sites a eu lieu afin de présenter la recherche et de discuter des thèmes à inclure dans le questionnaire.

(7) Parmi les 237 sites répertoriés, $11 \%$ sont destinés à la population générale mais présentent certaines informations sur la santé des minorités sexuelles, 38,4\% sont des sites destinées spécifiquement aux personnes LGBT et 50,2\% émanent d'organismes consacrés au VIH/sida, qui pour la plupart ciblent la population générale, certains sites étant spécifiquement destinés aux HARSAH. D'autres sites s'adressent particulièrement aux jeunes, aux communautés culturelles, aux autochtones, aux familles, aux personnes vivant avec le VIH et aux professionnels de santé. 
leurs caractéristiques ${ }^{(8)}$. Un peu plus du tiers (72) des organismes contactés a complété en partie ou en tout le questionnaire entre les mois de novembre 2008 et de juillet 2009.

\section{Résultats}

\section{Caractéristiques des sites participants}

La distribution des 72 organismes ayant complété le questionnaire indique une représentation importante des sites provenant du Québec (35\%), suivis de l'Ontario (25\%), de la Colombie-Britannique (14\%), de l'Alberta (10\%), les autres régions étant moins représentées (entre 1,5 et $4 \%$ ). Par ailleurs, $10 \%$ des sites sont destinés à la population générale mais présentent certaines informations sur la santé des minorités sexuelles, $46 \%$ visent les populations LGBT dans leur ensemble ou des minorités spécifiques, $44 \%$ sont des sites d'organismes consacrés aux problématiques du VIH/sida qui ciblent pour la plupart, la population générale, quelques-uns s'adressant toutefois spécifiquement aux HARSAH ${ }^{(9)}$.

\section{Les fonctions et activités interactives}

Notre analyse montre que $91 \%$ des sites participants affichaient une adresse courriel sur le site permettant de communiquer en différé avec un intervenant, $34 \%$ proposaient un babillard, $26 \%$ un forum de discussion, $11 \%$ une adresse courriel spécifique pour l'utilisation de programmes de messagerie instantanée permettant de communiquer en direct avec un intervenant (par exemple, Live Messenger) et $6 \%$ un salon de clavardage intégré au site. Par ailleurs, 35 \% des organismes avaient créé un profil de l'organisme sur les nouveaux espaces de socialisation devenus très populaires tels que YouTube ou MySpace, etc., ce qui permet de rejoindre d'autres usagers que ceux qui visitent les sites des organismes.

\section{Thèmes de santé abordés}

Un premier portrait des thèmes relatifs à la santé privilégiés sur ces sites fait apparaître les tendances suivantes (tableau I). Ces thèmes sont associés à deux dimensions de la santé soit 1) la santé physique, mentale ou les habitudes de vie et 2) les relations interpersonnelles et sociales.

Les thèmes les plus communs sur les sites participants (près de $50 \%$ ou plus de réponses dans les questionnaires) portent sur l'homophobie, le bienêtre sexuel, l'usage des préservatifs, les ITSS, la discrimination et le

(8) Le questionnaire demandait de préciser plusieurs aspects du travail des organismes quant à leurs activités sur l'internet tels que les langues utilisées sur les sites, les sources d'informations pour les contenus en ligne, les thèmes reliés à la santé abordés, les objectifs spécifiques des activités déployées, les modèles théoriques sous-jacents au contenu des sites et des autres activités, les statistiques quant à la fréquentation des sites, les ressources humaines et financières consacrées à ces activités, les partenariats avec d'autres organismes, les avantages perçus de ce type de contenus tant pour les organismes que pour les usagers et les difficultés et les limites reliées à la gestion et au développement des activités en ligne.

(9) On note une distribution parfois inégale parmi les organismes participants, par comparaison à l'ensemble des sites recensés. Si les organismes communautaires (75\%), les regroupements et les coalitions (en vue de la défense des droits et lutte contre l'homophobie, par exemple) (8,3\%) et les cliniques médicales sont assez bien représentés $(4,2 \%)$, d'autres types d'organisations sont moins présents dans l'échantillon tels que les centres communautaires $(1,4 \%)$ et les sites de socialisation et de rencontre $(1,4 \%)$. 
Tableau I : Distribution des problématiques touchant la santé sur les sites

\begin{tabular}{|c|c|}
\hline Homophobie & $54 \%$ \\
\hline Bien-être sexuel & $52 \%$ \\
\hline Usage des préservatifs & $52 \%$ \\
\hline Infections transmissibles sexuellement (agents pathogènes, symptômes, traitements) & $52 \%$ \\
\hline Discrimination & $52 \%$ \\
\hline Processus de dévoilement de l'orientation sexuelle (coming out) & $50 \%$ \\
\hline Relations familiales & $48 \%$ \\
\hline Vie de couple & $32 \%$ \\
\hline Violence dans une relation de couple & $28 \%$ \\
\hline Violence en général (verbale, physique ou sexuelle) & $28 \%$ \\
\hline Consommation problématique de drogues & $25 \%$ \\
\hline Suicide & $25 \%$ \\
\hline Dépression & $23 \%$ \\
\hline Fin d'une relation & $22 \%$ \\
\hline Consommation problématique d'alcool & $21 \%$ \\
\hline Décès d’un proche ou deuil & $20 \%$ \\
\hline Niveau de stress élevé/Anxiété & $19 \%$ \\
\hline Dépendance affective & $18 \%$ \\
\hline Image corporelle & $15 \%$ \\
\hline S'occuper d'un proche souffrant de maladie & $14 \%$ \\
\hline Trouver un partenaire stable ou régulier & $10 \%$ \\
\hline Sexualité compulsive & $8 \%$ \\
\hline Visionnement compulsif d'images érotiques (format papier, vidéo ou Internet) & $6 \%$ \\
\hline Dépendance à l'internet & $4 \%$ \\
\hline Dépendance au jeu & $4 \%$ \\
\hline
\end{tabular}

processus de dévoilement de l'orientation sexuelle (coming out) et les relations familiales. Près du tiers des sites traite de la vie de couple, environ un quart propose des contenus reliés à la violence, la consommation de drogues, le suicide et la dépression et un site sur cinq présente des contenus reliés à des thèmes tels que la fin d'une relation, la consommation d'alcool et le décès d'un proche ou le deuil. D'autres thèmes sont moins fréquemment abordés dans les sites (moins de $20 \%$ des organismes participants). Il s'agit des enjeux entourant les niveaux de stress ou d'anxiété, la dépendance affective, l'image corporelle, les soins envers un proche souffrant de maladie et la quête d'un partenaire stable ou régulier. La dépendance au jeu ou à l'internet ainsi que la problématique de la sexualité compulsive constituent également des thèmes minoritaires (moins de $10 \%$ des sites).

\section{Les interventions en direct}

L'analyse des résultats du questionnaire révèle que dix organismes (14 \%), provenant de l'Ontario et du Québec, ont développé des programmes 
d'interventions dans des sites de rencontre où l'on retrouve des salons de clavardage tels que Gay.com, Gay411, Squirt.org, Manhunt et Priape ${ }^{(10)}$. Ces initiatives s'organisent généralement autour d'une équipe d'intervenants et de bénévoles qui répondent en ligne et en direct aux questions des usagers à différents moments de la semaine.

Populations visées, sources d'informations pour les interventions en ligne et langue des échanges

Pour six des organismes, les HARSAH forment la principale population visée. Deux d'entre eux mentionnent cibler également les hétérosexuels. Quatre visent des minorités sexuelles comme les personnes transgenres, les queer, les hommes et les femmes allosexuels et deux ciblent les jeunes qui se revendiquent d'une orientation gaie ou d'une autre minorité sexuelle.

Sept organismes déclarent utiliser pour leurs interventions, les données scientifiques, les avis de médecins ou d'autres professionnels de la santé et les sources gouvernementales (par exemple, les directions de la santé publique ou les ministères). Six organismes interviennent en anglais, deux en français et deux sont bilingues. Deux organismes interviennent dans d'autres langues, l'un en cantonais et l'autre envisage de recourir à plusieurs langues (espagnol et langues africaines comme amharic et le swahili).

\section{Modèles théoriques, objectifs et enjeux éthiques}

Rares sont les organismes (2) qui appuient leurs interventions sur un modèle théorique. Les modèles mobilisés sont respectivement celui de la réduction des risques (11) et celui de la planification d'intervention écologique PRECEED-PROCEED (12).

Pour neuf organismes, ces interventions visent des objectifs spécifiques tels qu'offrir des informations à des populations difficiles à rejoindre autrement tout en respectant l'anonymat des usagers, sensibiliser les usagers aux thèmes reliés à la santé sexuelle (ITSS et VIH/sida, réduction des risques, lieux de dépistage), donner des réponses spécifiques à des questions non précisées dans la littérature et référer les usagers vers les ressources appropriées, tant à l'interne qu'à l'externe. Six organismes ont mentionné que ce type d'interventions se fondait sur des règles éthiques et de déontologie et trois d'entre eux les signalaient aux usagers.

\section{Formation des intervenants, ressources et partenariats}

Six organismes rapportent que leurs intervenants ont reçu une formation en intervention dont deux spécifiquement sur l'intervention en ligne. Ces formations portent sur des thèmes variés : les ITSS et le VIH/sida, les risques associés aux pratiques sexuelles, les traitements, le dépistage et d'autres

(10) Un organisme intervient dans un salon de clavardage intégré à un site destiné particulièrement aux jeunes gais, et deux ont un profil sur Facebook.

(11) La réduction des risques désigne l'ensemble des stratégies visant à limiter les risques et les dommages sanitaires ou sociaux associés à certaines pratiques [2].

(12) Le modèle de planification d'intervention écologique PRECEED-PROCEED [18] favorise la prise en compte des facteurs prédisposant, facilitant ou renforçant l'adoption et le maintien de comportements de santé tels que la sexualité à moindre risque. 
thèmes reliés à la santé sexuelle. Deux organismes offrent également une formation sur la démystification de l'orientation sexuelle ou la prévention du suicide.

Les ressources humaines (nombre des intervenants payés ou bénévoles) mobilisées dans le cadre de ces interventions sont très limitées (13). Un seul des organismes mentionne détenir un budget spécifique pour son site et ses interventions dans les salons de clavardage. Trois organismes ont développé des partenariats pour offrir ces interventions en ligne (gestion des horaires, conditions d'accès pour les intervenants, coordination des présences en ligne entre plusieurs organismes à l'échelle provinciale).

\section{Statistiques de fréquentation et thèmes des échanges avec les intervenants}

Le nombre d'interventions réalisées par chacun des organismes au cours du mois précédant l'enquête variait entre 10 et 60 (moyenne de 25) et entre 80 et 950 (moyenne de 172) au cours de la dernière année (14). Pour trois organismes, ce nombre est demeuré stable au cours de la dernière année et pour deux, il a augmenté. Pour sept organismes, la provenance des usagers est majoritairement locale dans des proportions variant de 60 à $100 \%$. Un seul organisme a indiqué que des usagers provenaient de leur province (30\%) ou d'autres pays (50\%) ${ }^{(15)}$.

Les niveaux de risques associés aux pratiques sexuelles (sexe oral en particulier), les ITSS et leurs symptômes et les services de dépistages disponibles sont les thèmes les plus discutés, suivis par les questionnements sur l'orientation sexuelle, le processus de sortie ou l'homophobie.

\section{Avantages et inconvénients}

Plusieurs avantages associés aux interventions en ligne sont rapportés. Pour les organismes, ces stratégies permettent de communiquer avec des populations difficiles à rejoindre autrement et de les diversifier (jeunes, personnes vivant en milieu rural, au début du processus de dévoilement de leur orientation sexuelle), d'augmenter la visibilité de l'organisme, d'offrir une possibilité d'implication flexible pour les bénévoles, de consulter et fournir rapidement, lors de l'échange en ligne, des informations basées sur des données probantes et adaptées aux besoins spécifiques de l'usager, de donner la possibilité aux intervenants de travailler par moment à partir du bureau et de conseiller plusieurs personnes simultanément sur différentes fenêtres de clavardage privées.

Les organismes évoquent aussi plusieurs avantages pour les usagers, qui renvoient à des interventions facilement accessibles et disponibles en tout temps (dans le cas du babillard), à l'obtention d'une information exacte qui tient compte des orientations sexuelles, à l'augmentation de l'accès aux ressources de dépistage et au counseling prédépistage. L'intérêt de l'anonymat est souligné par plusieurs organismes, car comme le rapporte

(13) Un seul organisme dispose à la fois de deux employés à temps plein et de trois bénévoles. Deux autres organismes ont un employé affecté à ce travail avec un ou deux bénévoles. Trois organismes ont un seul employé affecté à l'intervention en ligne et deux seulement une équipe de trois et quatre bénévoles.

(14) La moitié seulement des organismes a fourni des statistiques concernant leurs interventions.

(15) Deux organismes ne disposaient pas de données au sujet de la provenance des usagers au moment de l'enquête. 
l'un des gestionnaires, "il conduit dans la plupart des cas à plus d'honnêteté et donc à des interventions plus précises " et "réduit les probabilités de discrimination relative à l'appartenance culturelle ou à l'orientation sexuelle".

Les organismes rencontrent également plusieurs obstacles et rapportent certains inconvénients associés à l'intervention en ligne. Mis à part le manque de financement spécifique et le temps limité dont peuvent disposer les intervenants pour ce mode d'interventions dans leur charge de travail déjà élevée, des difficultés propres à l'environnement et au déroulement des échanges sont mentionnées. Tout d'abord, durant les séances de travail, le nombre d'interventions est difficile à prévoir, et peut fortement varier (de zéro à plusieurs interventions simultanément), ces modulations pouvant générer de la frustration chez les bénévoles. Par ailleurs, l'absence de signaux non verbaux peut rendre la communication plus difficile avec les usagers, entraîner des quiproquos lors des échanges et nécessiter des demandes de précisions fréquentes. Les enjeux entourant le difficile établissement d'une relation de confiance avec les usagers sont aussi soulevés par les intervenants.

\section{Discussion}

Ces premiers résultats exploratoires mettent en évidence la diversité des régions géographiques des organismes, des publics cibles, des stratégies de communication et d'intervention et des thèmes de santé discutés dans les sites orientés vers les populations LGBT. Ils soulignent l'importance du rôle des organismes communautaires et de prévention du VIH/sida ainsi que des regroupements/coalitions dans la structuration de l'espace santé canadien lié à l'internet et orienté vers les minorités sexuelles. Ces organismes contribuent de façon disproportionnée à assurer le transfert de l'information et les interventions auprès de populations avec lesquelles ils sont fortement impliqués, les sites provenant généralement des associations directement interpellées par les problématiques de la santé des LGBT. La distribution géographique montre la prédominance des régions comme le Québec, l'Ontario et, à un moindre degré, la Colombie-Britannique, ce qui reflète la concentration des minorités sexuelles dans les zones urbaines de Montréal, Toronto et Vancouver.

Concernant les modes d'intervention, les réponses aux questionnaires indiquent que la presque totalité des organismes recourt à l'adresse courriel, le moyen le plus simple pour échanger avec les usagers. De même, les médias sociaux sont utilisés pour diffuser de l'information par nombre d'organismes, l'efficacité de ce type de stratégie pour toucher d'autres usagers et augmenter ainsi la fréquentation sur les sites des organismes et institutions œuvrant dans le domaine de la de santé publique est d'ailleurs de plus en plus documentée. Par contre, ces médias sont peu utilisés pour réaliser des interventions en tant que telles, alors que la littérature documente aussi leur potentiel $[4,17]$. Ainsi, le recours à d'autres outils comme un salon de clavardage en direct, notamment lorsque celui-ci est intégré dans le site, est bien moins répandu dans les organismes. Cela s'explique sans doute par les difficultés associées au financement, aux 
développements informatiques requis et à la gestion mais aussi à l'expertise qu'exige ce type d'activité interactive, comme le suggèrent des travaux de recherche $[12,26]$. L'utilisation des babillards et des forums de discussions, est par contre très répandue parce que, entre autres, ce sont des outils interactifs existant depuis plus longtemps, que l'on peut se procurer gratuitement sur l'internet et qui sont faciles à insérer sur un site. Rares sont en effet les organismes qui possèdent des ressources véritablement formées aux technologies Web plus élaborées.

La distribution des thèmes de santé abordés sur les sites des organisations met, quant à elle, en évidence la place centrale des préoccupations face à l'homophobie, la discrimination et les processus de dévoilement de l'orientation sexuelle (coming out), qui contribuent à des problèmes sérieux de santé mentale $[5,8]$. Ces thèmes rejoignent, dans l'espace public contemporain, les revendications liées à l'accession à la pleine égalité citoyenne, indépendamment de l'orientation sexuelle. D'autres motifs retiennent aussi l'attention comme les références au bien-être sexuel et aux inquiétudes entourant la question de la transmission mais aussi de la prévention des ITS et du VIH/sida, ce qui reflète le retentissement de cette épidémie sur les minorités sexuelles, en particulier les HARSAH, mais aussi sur les organismes ciblant ces populations, nombreux à s'organiser autour de ces questions. Les enjeux entourant les relations familiales sont aussi des thèmes prévalents qui révèlent les résistances à l'acceptation des identités et orientations des LGBT par l'entourage immédiat. Les problématiques touchant la relation de couple et la violence interpersonnelle ou sociale sont aussi significatives, alors que celles liées à la santé mentale (drogues, suicide, dépression, consommation d'alcool) sont moins soulignées dans ces sites, une situation qui ne semble pas refléter les tendances épidémiologiques rapportées dans les recherches $[29,27,9,24,25]$. Cet écart demanderait à être explicité mais il suggère que les organismes sont sans doute peu outillés pour aborder ces problématiques en ligne, compte tenu de leur complexité. On constate également une faible présence des thèmes sur la compulsion et la dépendance face à la sexualité et à l'internet, des problématiques controversées dans la littérature psychosociologique [11].

\section{Conclusion}

Cette étude exploratoire portant sur les activités réalisées à l'aide de sites internet orientés vers les minorités sexuelles démontre l'importance de cet espace investi par de nombreux organismes communautaires qui fournissent un ensemble d'informations sur les enjeux de santé dont certains font aujourd'hui l'objet d'une vive attention. Ceux-ci reflètent clairement les préoccupations majeures entourant le statut problématique des minorités sexuelles dans la société contemporaine de même que les répercussions sur la santé du VIH/sida et des ITS dont la prévention constitue un enjeu crucial. D'autres thématiques pourtant significatives au plan social restent néanmoins encore peu explorées. Quant aux programmes d'intervention ciblés qui font appel à une interactivité internaute-intervenant, ils sont encore très peu nombreux et se heurtent à de nombreuses difficultés de développement, bien que leurs avantages soient reconnus. Les écueils liés à 
l'obtention de fonds pour le développement des interventions et la formation des intervenants jouent un rôle important, tout comme les contraintes communicationnelles qu'impose ce type d'inter-actions. Ces premiers résultats demanderont à être confirmés sur une plus grande échelle mais ils indiquent que le cyberespace constitue une dimension incontournable pour mieux comprendre, mais aussi pour mieux agir, sur les déterminants de la santé, notamment par la transmission des informations et les interventions en ligne.

\section{BIBLIOGRAPHIE}

1. Agence de la santé publique du Canada. Actualité en épidémiologie sur le VIH/sida. Division de la surveillance et de l'évaluation des risques, Centre de prévention et de contrôle des maladies infectieuses, Ottawa 2007.

2. Alan Marlatt G. “One”. In: Alan Marlatt G. Harm Reduction: Pragmatic Strategies for Managing High-Risk Behaviors. Guilford Press 2002: 3 p.

3. Banks C. The Cost of Homophobia: Literature Review on the Human Impact of Homophobia In Canada, Rochon Associated Human Resource Management Consulting Inc 2003.

4. Beard L, Wilson K, Morra D, Keelan JA. Survey of Health-Related Activities on Second Life. J Med Internet Res 2009; 11 (2): e17. URL http://www.jmir.org/2009/2/e17/

5. Berg MB, Mimiaga MJ, Safren SA. "Mental health concerns of gay and bisexual men seeking mental health services". J Homosexual 2008; 54 (3): 293-306.

6. Blais M, Dumas J, Lévy JJ, Ryan B, Thoër C, Léobon A. « Les programmes Internet destinés aux minorités sexuelles au Canada ». Congrès international de sociologie, Istanbul, Turquie, juillet 2008.

7. Bull S. «Internet and Other Computer Technology-Based Interventions for STD/HIV Prevention ». In: Edgar T, Noar SM, Freimuth VS (eds.). Communication Perspectives on HIV/AIDS for the 21st Century, Lawrence Eribaum Associates, New York 2007: 212-227.

8. Burgess D, Lee R, Tran A, van Ryn, M. "Effects of perceived discrimination on mental health and mental health services utilization among gay, lesbian, bisexual and transgender persons". J LGBT Health 2007; 3 (4): 1-14.

9. Cochran SD, Sullivan JG, Mays VM. "Prevalence of mental disorders, psychological distress, and mental health services use among lesbian, gay, and bisexual adults in the United States". I Consult Clin Psychol 2003; 71: 53-61.

10. Commission des droits de la personne et des droits de la jeunesse. De l'égalité juridique à l'égalité sociale vers une stratégie nationale de lutte contre l'homophobie. Rapport de consultation du Groupe de travail mixte contre l'homophobie 2007.

11. Cooper A, Delmonico DL, Burg R. "Cybersex Users, Abusers, and Compulsives: New Findings and Implications". In: The Dark Side of the Force, sous la dir. de A. Cooper, Philadelphy: Brunner-Routledge 2000: 5-29.

12. Curotto A, Rebchook G, Kegeles S. Opening a virtual door into a vast real world:. Community based organizations are reaching out reaching out to at-risk MSM with creative, online programs. Centre for AIDS prevention studies, University of California 2003.

13. Destombes C. "Internet 2008: Interactivité et logique affinitaire ". Le Journal du sida, Association de recherche, de communication et d'action pour l'accès aux traitements (ARCAT), Paris 2008: $207 \mathrm{p}$.

14. Dumas J, Lévy JJ, Ryan B, Thoër C, Léobon A. Recension préliminaire des sites canadiens de promotion de la santé destinés aux minorités sexuelles. Document inédit. Groupe de recherche Sexualité et genre : vulnérabilité et résilience, Université du Québec à Montréal 2007.

15. Dumas J, Rousseau R, Mensah MN. Êtes-vous satisfait ? Peut-on faire mieux... autrement ? Rapport d'enquête auprès d'hommes ayant des relations sexuelles et affectives avec d'autres hommes sur l'approche globale de la santé et du mieux-être à Action Séro Zéro, Rapport de recherche. Montréal : Action Séro Zéro 2008.

16. Engler K, Frigault LR, Léobon A, Lévy JJ. “The sexual superhighway revisited: A quantitative analysis of gay men's perceived repercussions of connecting in Cyberspace”. J Gay Lesb Soc Serv 2005; 18 (2): 3-37.

17. Gosselin P, Poitras P. Use of an Internet "Viral" Marketing Software Platform in Health Promotion. J Med Internet Res 2008; 10 (4): e47. URL: http://www.jmir.org/2008/4/e47/

18. Green LW, Kreuter WM. Health promotion planning; An Educational and Ecological Approach, 3rd edition. Mayfield Publishing Compagny, Mountain View, CA. 1999: 621 p. 
19. Hart SL, Bowen DJ. Sexual orientation and intentions to obtain breast cancer screening. J Womens Health (Larchmt) 2009; 18 (2): 177-185.

20. Institut national de la santé publique du Québec. Programme de surveillance de l'infection par le virus de l'immunodéficience humaine (VIH) au Québec, Ministère de la Santé et des Services sociaux, Laboratoire de santé publique du Québec 2007.

21. Kalichman SC, Cain D, Cherry C, Pope H, Eaton L, Kalichman MO. "Internet use among people living with HIV/AIDS: Coping and health-related correlates”. AIDS Patient Care ST 2005; 19 (7): 439-448.

22. Lévy JJ, Julien D et coll. Homosexualités : variations régionales, collection Santé et société, Presses de l'Université du Québec 2007.

23. Mehra B, Merkel C, Peterson Bishop A. The Internet for empowerment of minority and marginalized users. New Media Soc 2004; 6 (6): 781-802.

24. Meyer IH. "Prejudice, social stress, and mental health in lesbian, gay, and bisexual populations: Conceptual issues and research evidence". Psychol Bull 2003; 129 (5): 674-697.

25. Meyer IH, Northridge ME. (eds.). The Health of Sexual Minorities: Public Health Perspectives on Lesbian, Gay, Bisexual and Transgender Populations. Springer publications, New York 2007.

26. Noar SM, Clark A, Cole C, Lustria MLA. "Review of interactive safer sex Web sites: Practice and potential”. Health Commun 2006; 20 (3): 233-241.

27. Omoto AM, Kurtzman HS (eds.). Sexual orientation and mental health: Examining identity and development in lesbian, gay and bisexual people. Washington, DC: APA Books, 2006.

28. ONU Sida VIH et sexualité entre hommes: politique générale, programme commun des nations unies sur le $\mathrm{VIH} /$ sida 2006.

29. Paul JP, Catania J, Pollack L, Moskowitz J, Canchola J, Mills T, Binson D, Stall R. "Suicide Attempts Among Gay and Bisexual Men: Lifetime Prevalence and Antecedents", Am J Public Health 2004; 92 (8): 1338-1345.

30. Pew Internet and American Life Project. Health Information Online. Rapport de recherche 2005.

31. Réseau Québécois d'Action pour la santé des femmes. Pour le dire, rendre les services sociaux et les services de santé accessibles aux lesbiennes, RQASF 2003.

32. Ruf M, Lovitt C, Imrie J. "Recreational drug use and sexual risk practice among men who have sex with men in the United Kingdom". Sex Transm Infect 2006; 82 (2): 95-97.

33. Ryan B, Brotman $S$, Rowe $B$. Accès aux soins de santé : réflexions sur la santé et le bien-être des personnes gaies, lesbiennes, bisexuelles et bispirituelles au Canada. École de service social, Université McGill et le Centre d'études appliquées sur la famille, avec la collaboration d'EGALE (Equality for Gays and Lesbians Everywhere) 2000.

34. Shankle MD. The Handbook of Lesbian, Gay, Bisexual, And Transgender Public Health: A Practitioner's Guide to Services. Haworth press 2006.

35. Statistique Canada. Enquête sur la santé dans les collectivités canadiennes, 2003. URL http:// www.statcan.ca/Daily/Francais/040615/q040615b.htm.

36. Tang H, Greenwood GL, Cowling DW, Lloyd JC, Roeseler AG, Bal DG. "Cigarette smoking among lesbians, gays, and bisexuals: How serious a problem"? Cancer Cause Control 2004; 15 (8): 797-803.

37. Tjepkema M. Utilisation des services de santé par les gais, les lesbiennes et les bisexuels au Canada. Composante du produit no 82-003-X, Rapports sur la santé au catalogue de Statistique Canada 2008.

38. Trocki KF, Drabble L, Midanik L. "Use of heavier drinking contexts among heterosexuals, homosexuals and bisexuals: Results from a National Household Probability Survey”. J Stud Alcohol 2005; 66 (1): 105-110. 